\title{
Apparent motion of stimuli presented stroboscopically during pursuit movement of the eye*
}

\author{
ARNOLD E. STOPER \\ California State University, Hayward, California 94542
}

\begin{abstract}
In normal illumination, retrograde motion of the background (the Filehne illusion) can be seen during ocular pursuit, in contrast to stability seen during a saccade. In the present experiment, two stimuli were presented sequentially: (I) at disparate physical locations such that the pursuit movement of the eye caused them to excite the same retinal location, (II) at the same physical location, with pursuit movement causing disparate retinal excitations, and (III) with stationary fixation but with disparate physical locations such that the retinal excitation was identical to that of Condition II. Optimal movement was never reported for Condition I but was reported with essentially equal frequency in Conditions II and III. These results indicate a failure of compensation for pursuit movement, as does the Filehne illusion. The nature of the pursuit extraretinal signal was discussed, and it was argued that a distinctly different extraretinal signal is necessary for perceived stability during the saccade.
\end{abstract}

Interest in the subject of motion perception has recently been concentrated on the coding of image motion with respect to the retina; physiological mechanisms which seem capable of this coding have been discovered in the mammalian visual system (Hubel \& Wiesel, 1962; Barlow \& Hill, 1963). It is well known, however, that motion can be experienced without such image motion. If, for example, a smoothly moving luminous object is accurately pursued by the eye in a dark room, the motion of this object will be seen even though image motion with respect to the retina may be virtually eliminated. ${ }^{1}$ The generally accepted explanation of this perceived motion involves some process which "takes into account" the motion of the eye itself.

The existence of a "taking-into-account" process was proposed over 100 years ago by Helmholtz (1866), and its hypothetical functions have recently been clearly stated by Gregory $(1958,1966)$. Gregory postulates two distinct systems for the perception of motion. One system is responsible for detecting image movement with respect to the retina and is presumably mediated by low-level detectors such as those found by Barlow and Hubel and Wiesel; Gregory calls it the "image/retina system." The other system is responsible for detecting

\footnotetext{
*The research presented here is a portion of a doctoral dissertation presented to Brandeis University. It was performed during the tenure of an NIMH predoctoral fellowship, No. MH-22, 832-03. It was supported in part by Project MAC, an M.I.T. research program sponsored by the Advanced Research Projects Agency, Department of Defense, under Office of Naval Research Contract NONR-4102(01). I wish to thank Ulric Neisser for his guidance and encouragement throughout the course of this study. I also wish to thank John Senders, Ricardo Morant, Paul Kolers, and Samuel McLaughlin for their suggestions and comments. Thanks are also due Seymour Papert of Project MAC, for his advice and for making available the computer used for this research. and the members of Project MAC, who provided assistance in programming.
}

motion of an object which is followed by the eye. It works by taking into account movements of the eye; Gregory calls it the "eye/head system." The nature of this taking-into-account process is examined in this paper.

Traditionally, several other motion phenomena associated with eye movements have been explained by means of some form of taking eye movements into account. In addition to (1) the perceived motion of a pursued object, these are (2) the perceived stability of a scene even though voluntary saccadic eye motions cause its image to move over the retina, (3) the apparent motion of a stationary scene when the eye is moved "passively," i.e., by finger pressure, (4) the apparent motion of a stationary scene when a person with a paralyzed eye attempts, but fails, to move his eye, and (5) the apparent motion of an afterimage during voluntary saccadic movements.

The theorizing as to the nature of the process that takes eye movement into account has been directed mainly at an explanation of perceived stability (Phenomenon 2 above). Almost all theories (Helmholtz 1866; Hering, 1879; James, 1890; Sherrington, 1918; Koffka, 1935; Bruell \& Albee, 1955; Walls, 1951; von Holst, 1954; Rock \& Ebenholtz, 1962; Mack \& Bachart, 1969) seem ' to agree that image motion produced by saccadic eye movements somehow is cancelled by the taking-into-account process. ${ }^{2}$ These theories will be referred to here as cancellation theories. after Gregory (1958). The major theoretical controversy has been over the source of information as to the extent of eye movements. The proponents of the "intlow" version of the cancellation theory (James, 1890: Sherrington, 1918) assumed that this information flowed inwardly to the brain from stretch receptors in the eye muscles. while proponents of the outflow version of the cancellation theory (Helmholtz, 1866; von Holst. 1954) assumed that this information came 


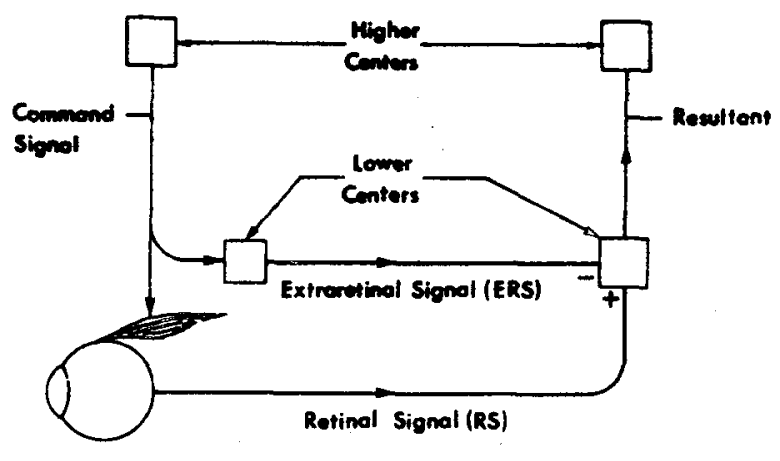

Fig. 1. A model of the cancellation theory (following von Holst, 1954).

from the command signal sent out from the brain to the eye muscles. The outflow version of the theory is generally accepted (Teuber, 1957; Howard \& Templeton, 1966) because of the difficulty the inflow version has in explaining Phenomena 3 and 4 above. The term "cancellation theory" in the following will refer to the outflow version only. A modern conceptualization of the cancellation theory, essentially due to von Holst, is shown in Fig. 1. Figure 1 can be taken to represent any of the cancellation theories referred to above. ${ }^{3}$ These theories share the following critical assumptions.

(1) Given the extent of a particular eye movement, the magnitude and direction of image motion resulting from that eye movement can be predicted by the visual system. As represented in Fig. 1, information as to the extent of predicted image motion is carried by the magnitude and sign of the extraretinal signal (ERS).

(2) The predicted motion is somehow summed algebraically with the actual image motion over the retina. As represented in Fig. 1, information as to the magnitude and direction of the actual image motion is carried by the magnitude and sign of the retinal signal (RS). The ERS is, in effect, subtracted from the RS.

(3) The resultant of this algebraic summation of the two signals determines the magnitude and direction of the perceived motion. In Fig. 1, a "lower center" produces the summation of signals and sends the resultant up to "higher centers" (terminology following von Holst, 1954).

These assumptions are implicit in all cancellation theories, even though terminology may differ greatly. The signal that carries information as to the predicted extent of the image movement has been variously called: the sense of innervation or effort of will, by Helmholtz (1866); the change in retinal local sign (see Note 3), by Hering (1879); the efferent copy, by von Holst (1954); and the corollary discharge, by Teuber (1957). The term "extraretinal signal" (ERS) is used here, following Matin et al (1969). The ERS would presumably be generated by what Gregory calls the "eye/head system," while the RS would be generated by what he calls the "image/retina system" (but see Note 3). Many theorists do not explicitly speak of a signal carrying information as to predicted image movement. but such a signal is implied by the use of such terms as "taking eye motion into account." Some versions of the theory (e.g., Teuber, 1957; Gregory. 1966) refer to a "comparator" which produces a signal proportional to the difference between the ERS and the RS, but this process is essentially also a process of algebraic summation of the two signals.

The assumption of algebraic summation of the ERS and the RS is perhaps the most attractive feature of the cancellation theory. It is this assumption which allows the parsimonious explanation of all five eye-movement phenomena listed above by means of a single hypothesized extraretinal signal. Because of the assumed algebraic summation, this signal can have a dual function: (i) It can reduce the magnitude of perceived motion by antagonism to the RS. If equal in magnitude to the RS, as in a normal eye movement, it will cancel it completely, resulting in perceived stability (Phenomenon 2). (ii) If uncancelled, it has the same capacity as the RS to generate the perception of motion. If, for example, the RS is absent, the resultant percept of motion will be equal to the ERS. This is presumably the case in Phenomena 1,4 , and 5.

If the eye is stationary or moved passively, the ERS is presumed to be absent. Any RS produced by image motion will thus proceed uncancelled to "higher centers" to produce the perception of motion. This is presumably the case when viewing a moving object with a stationary eye, or a stationary scene with passive eye motion (Phenomenon 3).

The assumption of a unitary ERS to explain all five of the eye-movement phenomena listed above will be questioned in this paper. In particular, the assumption that the phenomenon associated with pursuit movement (Phenomenon 1) is due to the same ERS that is responsible for the phenomena associated with saccadic movement (Phenomena 2, 4, and 5) will be challenged. This assumption is made explicitly by Koffka (1935), Bruell and Albee (1955), Walls (1951), Rock and Ebenholtz (1962), Gregory (1958), and Mack and Bachant (1969), and implicitly by many others. It has long been known (Dodge, 1906), however, that the pursuit is a distinctly different eye movement from the saccade, and recently gathered evidence (Rashbass, 1967) indicates that different brain centers may be involved in the control of each type of eye movement. It might, therefore, be suspected that the ERS that accompanies the pursuit has a different nature from the ERS that accompanies the saccade. I will attempt, in this paper, to present evidence for this difference and to examine the nature of the pursuit ERS.

\section{The Filehne Illusion}

If the ERS which produces the perceived motion of a pursued object has the characteristics assumed by the cancellation theory. it should cancel whatever image 
motion over the retina is produced by the pursuit movement itself. It can be readily demonstrated, however, that this cancellation does not occur under normal conditions.

If, in a fully lighted room, an $\mathrm{O}$ follows a smoothly moving object (he can move a pencil or his fingertip), he may notice the background moving in the opposite direction to the object. This illusion was first reported by Filehne (1922), who considered that it was an explanation of the Aubert-Fleischl phenomenon. Others (Gibson, 1954; Gregory, 1958), since then, have noticed it but, in general, it has not been of great concern to theorists.

It is surprising that more attention has not been paid to this phenomenon. One reason may well be the difficulty many Os have in seeing the illusion. In my own experience, perhaps only $50 \%$ of naive Os report background movement in the conditions described above. This difficulty appears to be in part due to the difficulty of attending to the background while at the same time fixating the moving object. Another reason some Os may not report the illusion is the paradoxical nature of the motion perception involved. Most Os agree that background moves but that it doesn't seem to change position (see Note 6). This motion can perhaps best be described using Gibson's (1950) terminology as motion of the "visual field" while the "visual world" seems to remain stable.

The perceived motion of the visual-field background during pursuit may be contrasted with perception during the saccade. In the latter case, under normal circumstances, the eye movement produces image movement over the retina, but no hint of any visual-field movement is seen. How can we account for this difference in perception between the two types of eye movement? It has been suggested by Gregory (1958) that the failure of cancellation during pursuit movement is due to the nature of the motion of the background image. During pursuit, he points out, the background image is "dragged" over the retina, while during the saccade, the image "hops," i.e., it moves discretely from one position to the next. The saccadic movement is so fast, about $600 \mathrm{deg} / \mathrm{sec}$ (Westheimer, 1954a), that for all practical purposes the image is absent during its transition. The image motion during a saccade thus resembles the stimulus presentation for stroboscopic movement; if there were no extraretinal cancelling signal, the apparent movement would be stroboscopic in origin. According to Gregory, the ERS during the pursuit is similar to that during the saccade, but, he says, although capable of cancelling a "hopping" signal, it is for some reason incapable of cancelling a retinal signal produced by an image "dragging" across the retina.

If the pursuit ERS is of the same nature as the saccadic ERS. it should be capable of cancelling a retinal signal produced by an image "hopping" across the retina. Condition II of the experiment described below is designed to test this assumption; in this condition. a stimulus is flashed twice in the same physical location, but pursuit movement of the eye causes the two stimuli to excite two distinct retinal locations, i.e., the image "hops" across the retina. According to Gregory's assumption, this image motion should be cancelled.

An additional factor in the design of this experiment was ease in comparison of perception during the pursuit movement with perception during the saccade. Only pursuit movements were investigated in this report; the analogous saccadic data were obtained by Rock and Ebenholtz (1962) in an investigation of stroboscopic motion. Rock and Ebenholtz were concerned with showing the importance of phenomenal (experienced) location of stimuli over anatomical location; one means they used to separate the two was to have the $S$ perform saccadic eye movements between two positions in space. In their Experiment I, Part I, the apparatus was arranged so that one stimulus excited the S's fovea when his eye was in Position 1 and the second stimulus excited his fovea when his eye was in Position 2. Despite the fact that the two stimuli excited the same retinal location (the fovea), the Ss saw them located in different phenomenal positions and reported apparent movement between the two stimuli. This result can be taken as a demonstration that Phenomenon 5, the apparent motion of an afterimage in a dark room, occurs with brief and discrete presentation of stimuli. Our Condition $I$ is designed to be similar to Part I of Rock and Ebenholtz, but with $S$ performing pursuit rather than saccadic eye movements. Part Il of the Rock and Ebenholtz experiment was the converse of Part I. Here, Stimuli I and II were in the same place in physical space, but the saccadic movement of the S's eye caused them to excite different retinal locations. Nevertheless, the Ss reported the perception of a stationary light flashing; i.e., they saw no apparent motion. This result can be taken to be a demonstration that Phenomenon 2, the perceived stability of a scene despite saccadic eye movements, occurs with brief and discrete presentation of stimuli. Our Condition II is designed to be similar to Part II of Rock and Ebenholtz, but with pursuit rather than saccadic eye movements.

\section{METHOD}

\section{Apparatus}

A PDP-6 computer (manufactured by the Digital Equipment Corporation) was used to display all stimuli and record and tabulate all responses. In addition to the CRT display unit (Type 340), an arithmetic processor (Type 166) was used to control the display and perform the necessary calculations for changing the display with time.

The display area consists of a 1.024 by 1.024 point matrix. Any one of over a million diserete points can be displayed at any given time. In the appropriate mode. each point requires only a 1.5 -microsec plotting time. Thus. approximately 600 separate points can be displayed in $1 \mathrm{msec}$. permitting a reasonably. complex scene to be presented with apparent continuity. The total display area was $.25 \times .25 \mathrm{~m}$.

The cathode ray tube phosphor was Type P7, which has two 


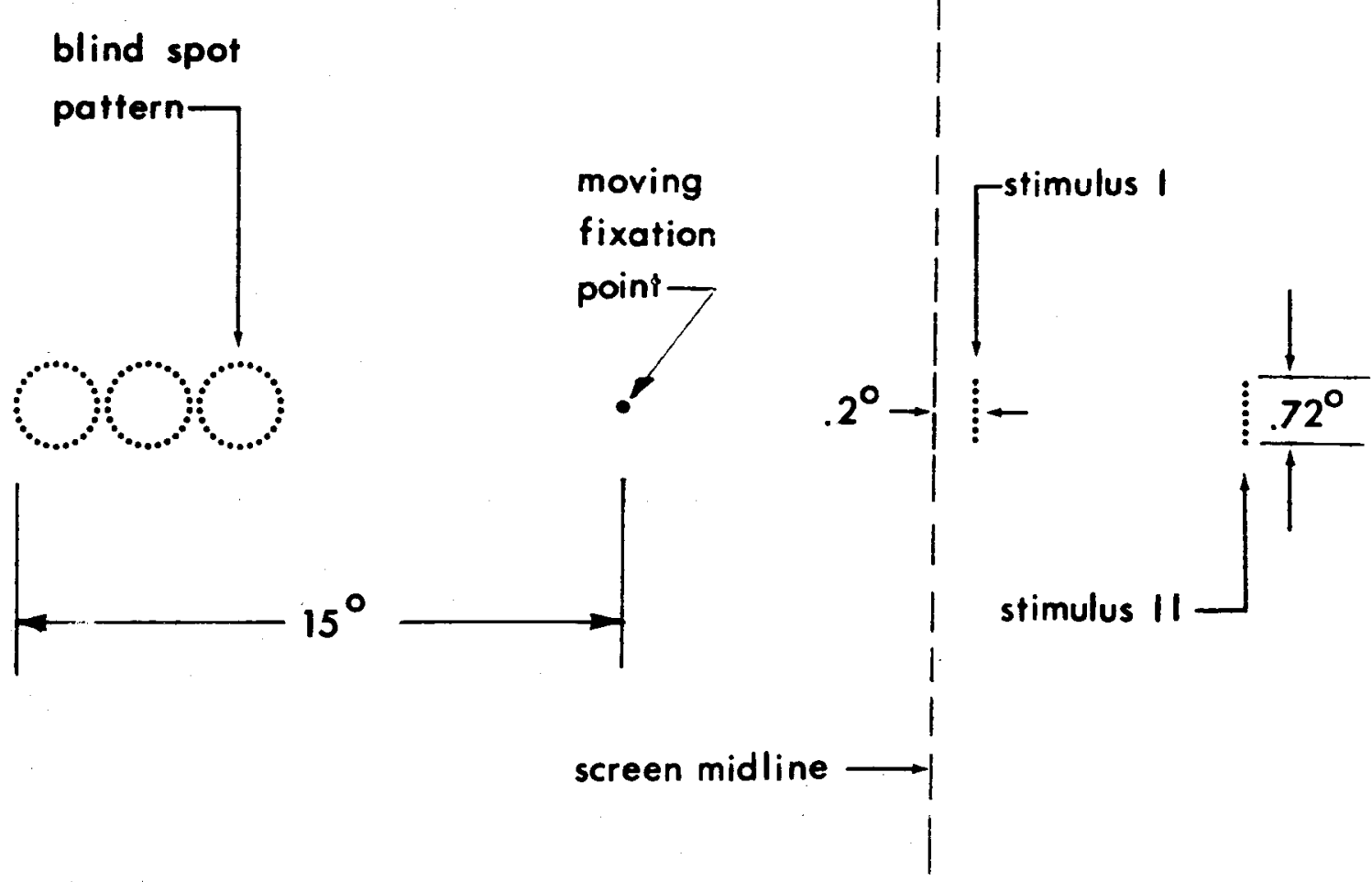

Fig. 2. Elements of the visual display. The screen midline was not displayed. The relative positions of Stimuli I and II shown are those for Condition I; the distance between stimuli depends on the time interval. For Condition II, Stimulus II is superimposed on Stimulus I; for Condition III, Stimulus II is presented to the left of Stimulus I.

distinct components. One component has an extremely short persistence (specified to decay at 60 microsec to $10 \%$ of initial value) and is blue in color (peak intensity at $4,400 \AA$ ). The other component has a much longer persistence, but it is predominantly yellow in color (peak intensity at $5,500 \AA$ ). It was desired, in this experiment, to present flashes of as short a duration as possible; therefore, the scene was viewed through a deep blue filter (Wratten 47B), which removed virtually all of the long-persistence component.

The responses of the $S$ were input to the computer by means of a "joystick" control, which was operated by the E. Three distinct responses were possible with this device; these responses were recorded and tabulated by the computer program. Any response automatically initiated the next trial.

The time of the various stimuli displayed was controlled by a real-time clock using a crystal oscillator standard, accurate to approximately one part in $10^{5}$.

\section{Visual Presentation}

All viewing was done monocularly with the left eye. A viewing hood was mounted on the display unit, and a black cloth was draped over the S's head to eliminate all extraneous light. The S wore goggles, in which were mounted the viewing filter. These goggles were held stationary against the surface of the viewing hood, eliminating virtually all head movement. Viewing distance was $50 \mathrm{~cm}$.

The elements of the visual scene used in the experiment are shown in Fig. 2. In Conditions I, II, and IV, the fixation point appeared at the left edge of the screen and moved steadily to the right, at a constant velocity, until it reached the right edge and disappeared. The total extent of travel of the fixation point was $27 \mathrm{deg}$. The $\mathrm{S}$ was instructed to follow this point as accurately as possible. At the moment this fixation point reached the precise midline of the screen, Stimulus I was flashed. The location of
Stimulus I was in all cases $.2 \mathrm{deg}$ to the right of the midline of the screen and at a vertical level such that the horizontal path of the fixation point bisected the stimulus. Stimulus II was flashed at a preselected time after the occurrence of Stimulus I, at a variable horizontal distance from Stimulus I, dependent on this time and on the experimental conditions.

\section{Eye-Motion Control}

The "blind-spot pattern" illustrated in Fig. 2 was used as an eye-motion control.4 At the start of each session, it was adjusted so that its image fell on the blind spot of each $S$ when he fixated the moving point. The pattern moved with the fixation points and, under normal circumstances, was never seen by the $S$ during a trial. The $S$ was instructed to reject the trial if this pattern ever became visible. This control served to indicate deviations of the eye from the fixation point only if the deviations were greater than about $2 \mathrm{deg}$. This was the case if the $S$ attempted to fixate a stationary stimulus instead of continuously following the moving point. The major function of this control was as a training device. A practiced $S$ rarely reported seeing the blind-spot pattern, but could usually see a slight glow (due to internal reflections etc.) in the area of this pattern. No inferences are made about the accuracy of the pursuit movement from the fact that the blind-spot pattern was not reported to be visible. The experimental results can be taken as evidence that $S s$ were, in fact, performing accurate pursuit movements.

\section{Display Characteristics}

The vertical lines (Stimuli I and II, Fig. 2) are, in fact, each composed of seven separate points. With the viewing conditions used, however, the lines appeared unbroken. These lines were $.72 \mathrm{deg}$ long. The fixation point was approximately $.03 \mathrm{deg}$ in diam, while the blind-spot pattern was 1.78 deg in length and 
$.72 \mathrm{deg}$ in height. The left edge of this pattern was approximately $15 \mathrm{deg}$ to the left of the fixation point.

The various elements of the display were at approximately the same apparent brightness. No exact measure could be made of stimulus brightness, due to the nature of the display.

The fixation point was moved by incrementing its position periodically in discrete steps. The velocity of the point was controlled by varying the period of the incrementation. The speed of $13.5 \mathrm{deg} / \mathrm{sec}$, for instance, resulted from an increment of approximately $.03 \mathrm{deg}$ occurring once every 2.04 microsec. The point was, in general, displayed for 200 microsec at the time of each incrementation and, in the above case, displayed at a rate of $500 \mathrm{~Hz}$. This was well above c.f.f., and the point always appeared to be on continuously and to move smoothly.

The exposure times of Stimuli $I$ and II are estimated to bo somewhat less than 1 msec: Each stimulus was actually repeated five times within this period. Each separate exposure took about 10 microsec and recurred at an interval of about 100 microsec. The five repetitions thus took less than 600 microsec, and the persistence characteristics of the phosphor inply that the stimulus had decayed to well below threshold $1 \mathrm{msec}$ after the initial exposure. The blind-spot pattern was presented at a rate of $10 \mathrm{~Hz}$. It was, of course, not displayed at all until the fixation point had reached the approximate midline of the screen (since it was positioned $15 \mathrm{deg}$ to the left of the fixation point, it was off the edge of the screen until this time).

\section{Subjects}

The Ss were three female employees of Project MAC. They had normal vision and were naive as to the purpose of the experiment, but were instructed as to the importance of accurate pursuit movements.

\section{Procedure}

The essential requirement of the $S$ was that he report "good" motion of a vertical line when it was seen. In all conditions of the experiment, the $S$ was given the following instructions regarding the apparent motion: "You will sometimes see two lines flashing on the screen simultaneously. Sometimes you will see one line flash, then a little later a second line. But sometimes you will see one line appear in one position and move to another position. Whenever this motion occurs, say 'yes.' Whenever you are not sure whether you actually saw a line moving or not, say 'no.'"

In Conditions I and II, the S was instructed to follow the moving fixation point with her eye as carefully as possible. She was given at least $5 \mathrm{~min}$ of practice in doing this and told that she should not see the pattern in the blind spot if she was, indeed, following accurately. She was constantly reminded of the difficulty of accurate following and that the task required great care. During the actual experiment, she was told to report any instance of the blind-spot pattern's appearing in her view. When this occurred (quite rarely), the trial was rejected and repeated immediately. The trial was also rejected if the $S$ failed to detect the line stimulus.

\section{Condition I}

Stimulus II was presented so as to excite the same retinal area as Stimulus I. This was accomplished by presenting both stimuli in the same position relative to the moving fixation point (stimuli were presented $.2 \mathrm{deg}$ to the right of the fixation point). This required that the physical location (location on display screen) of Stimulus II vary with time. Stimulus II was displayed at distance $v t$ to the right of Stimulus $I(v=$ velocity of fixation point, $t=$ time interval between Stimulus $I$ and Stimulus II).

\section{Condition II}

Stimulus II was presented at the same physical position as
Stimulus I (.2 deg to the right of the midline). Ten values of time interval were used, ranging from 20 to $350 \mathrm{msec}$. The time interval for each trial was selected randomly by the computer from a predetermined list. When each of the 10 time intervals had been used, a new series was begun. Ten such series were used, for a total of 100 trials for each $S$. The fixation point traveled at a velocity of $13.5 \mathrm{deg} / \mathrm{sec}$, from left to right. Because the $S$ followed the moving fixation point, the eye changed position between the time of Stimulus I and Stimulus II, and the two stimuli excited different retinal points. The distance between the excited points on the retina thus was dependent upon the time inveral, the distance being greater for a longer time interval.

\section{Condition III}

In this condition, the fixation point remained stationary at the center of the screen. The physical position of Stimulus II was varied with the time intervals in order to duplicate the retinal excitations of Condition II. Stimulus II was therefore displayed at a distance vt to the left of Stimulus I.

\section{Condition IV}

This condition was identical to Condition II, except for the addition of a stationary fixation point. The $S$ was instructed to fixate the stationary fixation point rather than the moving one, which was also visible.

\section{RESULTS}

Data from Conditions II and III are plotted in Fig. 3. No reports of optimal motion were ever made in Conditions I and IV. (When it became apparent with each $S$ that motion was rarely, if ever, reported, the condition was discontinued.) Thus, the full series of 100 trials per $\mathrm{S}$ was not run for Conditions I and IV.

\section{Condition I}

The physical presentation of the stimulus lines in Condition I would normally produce the experience of optimal stroboscopic apparent movement at certain time intervals; this is seen to be the case in Condition III, where physical presentation of stimuli is essentially identical (distance between stimuli is vt, but in opposite direction) to Condition I, but fixation is stationary. Good apparent motion was also reported in Part I of Rock and Ebenholtz (analogous to Condition I). In contrast, no $S$ ever reported optimal movement of the stimulus line in Condition I. Ss reported that at long time intervals between stimuli, the line could sometimes be seen moving as a unit with the moving fixation point, but this was a much weaker experience of movement than the optimal movement reported in Condition III. The experienced motion was seen only with difficulty. and no attempt was made to measure it.

Another difference between Conditions I and III was the absence of the "phenomenal mode of simultaneity" in Condition I. In classical stroboscopic motion experiments, the phenomenal impression at short time intervals (the time interval for motion depends on many factors: it is governed approximately by the classical "Korte's laws") is that the two stimuli seem to be 


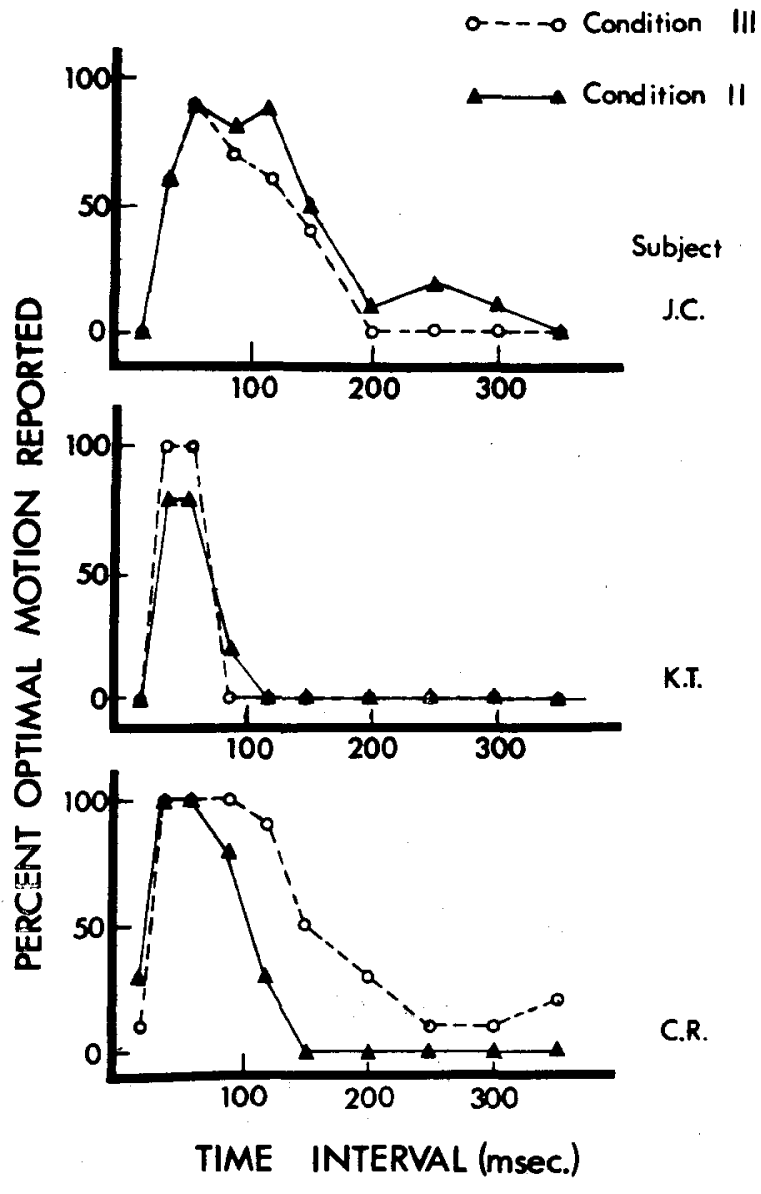

Fig. 3. Data of three Ss plotted separately. Conditions I and IV are not shown, as no optimal motion was reported for any time interval under these conditions.

flashed simultaneously, side by side, with no impression of motion between them. This was the case in Condition III for intervals below $40 \mathrm{msec}$, but not in Condition I. With moving fixation and the two stimuli striking the same retinal location, the side-by-side appearance of simultaneity never occurred: (This result could be taken as evidence that Korte's laws apply only for stationary fixation.)

\section{Conditions II and III}

In Condition II, even though the two stimuli were flashed in the same physical location, frequent apparent motion was reported. This motion was always in a direction opposite to the motion of the fixation point. As can be seen from Fig. 3, it occurs only for a limited range of time intervals.

Condition III duplicated the retinal excitation pattern of Condition II. The only difference between the two conditions insofar as the visual system is concerned is that in Condition II, the fixation point was perceived to move steadily to the right. The perceived motion of this point was presumably caused by the extraretinal signal accompanying the pursuit movement. We now ask whether the existence of this signal had any significant effect on the perception of the stroboscopic motion.

The total number of reports of optimal motion in Condition III was 104 out of 300 trials (as compared to 91 out of 300 in Condition II). The difference between the two conditions is not statistically significant $(\alpha=$ .128). One S (J.C.) reported more motion during Condition II than during Condition III. The ERS thus seems to have no significant effect on the perception of motion caused by retinally disparate stimuli.

An examination of the curves in Fig. 3 also shows that roughly the same time-distance parameters govern the motion seen in both Conditions II and III. For each S, the maximum percent of motion occurred at the same time interval, whether produced by physically separated or by physically superimposed stimuli. This result implies that the same mechanism was responsible for the perceived motion in both conditions.

\section{Condition IV}

The physical stimulus presentation was identical to Condition II, but the S kept his fixation on a stationary point. No $\mathrm{S}$ ever reported motion of the stimulus line under this condition. This result demonstrates that the simple presence of a moving fixation point is not sufficient to "induce" motion to the stimulus line; ocular pursuit of the moving point is necessary for the motion of the line to be seen.

\section{DISCUSSION}

\section{Does the Pursuit ERS Cancel the RS?}

The main result of this experiment is the frequent occurrence of optimal motion in Condition II. The pursuit ERS is evidently insufficient to cancel this motion. At the $60-\mathrm{msec}$ time interval, for example, optimal motion is reported in $90 \%$ of the trials despite the fact that two stimuli were flashed in the same physical location. This is a demonstration that the Filehne illusion occurs even 'with discretely flashed stimuli.

It may be argued that the apparent motion of the stimuli in Condition II was "induced" by the moving fixation point. One reason this argument is implausible is that the fixation point is much smaller than the vertical line that was seen in apparent motion; one of the primary rules governing induced motion is that it is the larger of two objects which induces motion to the smaller (Duncker, 1929; Wallach, 1959). Additional evidence against this argument is the result of Condition IV. Here, the moving fixation point was present, but, in the absence of actual pursuit movement of the eye, it had no detectable tendency to induce motion to the vertical line stimuli.

Condition II shows that the cancelling effect of the 
pursuit ERS was insufficient to prevent frequent occurrence of apparent motion. Was there any cancelling effect at all? Condition III differs from Condition II only in that the eye is performing a pursuit movement in Condition III but is fixated on a stationary point in Condition II. The retinal excitation conditions are identical. Any cancelling effect of the pursuit ERS would be evidenced in a diminished occurrence of stroboscopic motion in Condition III, but our results show the difference between the two conditions to be insignificant. Evidently, the pursuit ERS has no discernible cancellation effect on stroboscopic image motion under these conditions.

The Filehne illusion was presented above as evidence that the pursuit ERS fails to cancel movement of a continuously illuminated background. Gregory proposed that a cancelling signal does occur during pursuit movement as well as during saccadic movement, but that it does not cancel background movement during pursuit, because the background image "drags" across the retina; he implied that if it "hopped" across, as it does during the saccade, it would be cancelled. The results presented here show, however, that the pursuit ERS fails to cancel even stroboscopic movement, when the background image "hops" over the retina. The conclusion drawn is that a cancelling signal simply does not occur during pursuit movement. The pursuit ERS does occur, as evidenced by the apparent motion of the pursued object, but it is shown not to have any significant cancellation function.

\section{Comparison of Pursuit ERS with the RS}

An unexpected result was the complete lack of optimal motion reported in Condition I. It was expected that the flashed stimuli in this condition would be seen in motion even though they produced no image displacement over the retina; the moving fixation point itself, after all, was seen to be in clear and continuous motion, even though its image did not shift over the retina. The results of Condition I show, however, that the pursuit ERS that produced the perception of motion of the fixation point failed for some reason to produce the perception of motion of the briefly flashed vertical line stimuli. The reason for this failure is apparently the relatively brief ( $350 \mathrm{msec}$ or less) time that the vertical line stimuli were present. If vertical line stimuli are flashed repeatedly so that they excite the same retinal location but for a longer period of time (around 1 or $2 \mathrm{sec}$, cf. Stoper, 1967, p. 113), the phenomenal appearance is that of a smoothly moving vertical line. For the brief presentation used in Condition I, however, the apparent motion generated by the pursuit ERS was never strong enough to meet the criterion for optimal motion set by the instructions, i.e., that the line "looks as if it were moving from one place to another."

The faint impression of motion seen in Condition I stands in sharp contrast to nearly $100 \%$ ocqurrence of optimal motion at critical time intervals in Condition III. The physical stimulus presentation is essentially identical in the two conditions, being the stimulus presentation for the classic stroboscopic motion effect. In Condition I, however, the eye "follows" this motion, causing the two stimuli to excite the same retinal location and, thereby, greatly diminishing the strength of the motion. This contrast in motion perception seems to indicate some basic difference between the pursuit ERS and the retinal signal.

In normal viewing conditions, the pursuit ERS "mimics" the retinal signal (the latter must be the biologically more primitive of the two signals). This mimicry is good enough so that Os ordinarily do not distinguish between the motion seen when following a moving object from that seen when its image moves over the retina (Gibson, 1958). But the mimicry is not perfect, as was first demonstrated by Fleischl (1882). He found that phenomenal velocity of a continuously moving object was about twice as fast when observed with pursuit movement. This phenomenon is commonly known as the Aubert-Fleischl paradox. Using more controlled conditions, but also with continuously moving stimuli, Brown (1931) found an average velocity ratio of 1.43 to 1 , but with wide variations. With the briefly flashed stimuli used here, this difference between pursuit and stationary fixation is greatly exaggerated; it can no longer even be described as a difference in apparent velocity. A better description of the difference between Conditions I and III is that the motion seen in Condition III (via the retinal signal) was much more "insistent" or attention attracting than the faint motion of the vertical line seen in Condition I (via the pursuit ERS). Evidently, the difference between motion generated by the RS and that generated by the pursuit ERS is much greater with briefly presented stimuli than with a continuously moving stimulus. One possible explanation for this is that for the pursuit ERS to generate apparent motion, an image must be present long enough for the eye to move through some minimum angle. This angle would be much larger than the minimum image displacement over the retina needed to produce apparent motion. In other words, the eye/head system is less sensitive to displacement than the image/retina system. Nevertheless, the two systems normally produce signals which result in roughly equal judgments of apparent velocity.

\section{Interaction of Pursuit ERS and RS}

It was concluded above that the pursuit ERS and RS have no tendency to cancel each other. This means that there is no algebraic summation of the two signals; instead, they remain independent of each other. But what happens when the RS indicates an object is moving in one direction and the pursuit ERS indicates it is moving in the opposite direction? One possible resolution of this paradox is demonstrated in the 
well-known phenomenon of "motion analysis" (Duncker, 1929; Wallach, 1959; Johanssen, 1950). In one demonstration of this phenomenon, a wheel rolls in a dark room with one illuminated point at its rim; this point describes a cycloidal path, and this path is perceived. If now a light at the hub is added, the cycloidal motion is perceived as two distinct components: a circular motion of the rim point as it rotates about the hub and a translatory motion of the whole rolling wheel. Consider for a moment the normal case where the eye follows the translatory motion of the whole wheel (or the point at the hub). The point at the rim would in this case describe a circular path over the retina, and the retinal signal would contribute the circular component of the perceived motion. The pursuit ERS, on the other hand, would contribute the translatory component of the perceived motion. At certain times, however, these two components have opposite direction, as when the rim point reaches the bottom part of its orbit. At this time, it seems to move forward in its translatory component, since it is a part of the wheel which is seen translating as a whole, but backward in its circular component. Thus, though seemingly a paradox, a single object can be seen to move in two opposite directions at once; the pursuit ERS and RS can simultaneously contribute motion of opposite direction to the same object without cancelling each other. 5

Another resolution of this paradox is demonstrated in the Filehne illusion and in the present Condition II. Here the effect of the pursuit ERS is simply washed out wherever there is a retinal motion signal present. The pursuit ERS seems to produce a noticeable effect only when an image is stationary with respect to the retina, or, as in the case of motion analysis, when a complex moving pattern presents some global aspect which is stationary with respect to the retina.

\section{Pursuit ERS Compared to Saccadic ERS}

No good apparent motion was ever reported in Condition I; Rock and Ebenholtz (1962), however, found frequent good motion in their analogous saccadic condition (Part I). Frequent apparent motion was reported with retinal separation of stimuli in Condition II; Rock and Ebenholtz again found just the opposite, no apparent motion, with their analogous saccadic condition (Part II). The differences might be described by saying that Rock and Ebenholtz found good compensation for saccadic eye movement, while the only evidence of compensation for pursuit eye movement in the present study was the observed motion of the pursued fixation point. One possible explanation for these differences might be the differences in extent of the eye movements investigated. This was $18 \mathrm{deg}$ in the Rock and Ebenholtz study and variable in the present study but less than 2 deg for the critical time intervals. It is possible that only eye movements of some minimum extent (above $2 \mathrm{deg}$ ) are capable of generating apparent movement of a retinally stable image. whether the eye movement is pursuit or saccadic, and that the pursuit movements used here were simply too small to generate apparent motion in Condition I.

According to this explanation, apparent motion of a stimulus object moving with the retina would be seen only if its image were present long enough for the eye to move through some minimum angle. This condition would be met for the moving fixation point. and thus its motion is seen clearly. For the case of the saccade. informal observations suggest that apparent motion of an afterimage occurs only if the saccade is larger than some minimum angle, though no clear evidence on this matter seems to exist in the literature. It remains to be demonstrated that the minimum visual angle necessary to generate apparent motion is the same for a pursuit movement as for a saccadic movement.

While the small extent of the pursuit movement investigated could be the reason for failure of motion generation in Condition I, it is not plausible that it is the reason for failure of motion cancellation in Condition II. An explanation of failure of cancellation based on small extent must assume that cancellation would also fail for a 2-deg saccade. It is unlikely that this is the case, though once again clear evidence from the literature is lacking. Controlled studies of compensation during saccades of the order of $2 \mathrm{deg}$ have been done (Matin et al, 1969; Matin et al, 1970; Monahan, 1972), but the task of the $\mathrm{S}$ was to judge relative position rather than apparent motion. Monahan had Ss compare position of stimulus flashed before saccade with one flashed after, and found about 2-deg. compensation for a 2-deg eye movement. Unfortunately, he did not ask his Ss to report on apparent motion, and so we cannot directly compare his results to ours. It is possible to have a failure of motion cancellation even though position compensation is taking place, as evidenced by the Filehne illusion. ${ }^{6}$

Even in the absence of the appropriate controlled study, it seems safe to conclude, on the basis of motion cancellation during normal vision, that the results of Rock and Ebenholtz, Part II, can be generalized to saccades of the order of $2 \mathrm{deg}$. A 2-deg saccade is a common occurrence in normal vision; the success of motion cancellation is evidenced by the fact that apparent motion of viewed stationary objects is virtually never seen. There seems to be no reason to assume that Rock and Ebenholtz would have had different results if they had studied smaller saccades. The failure of cancellation in the present Condition II cannot, therefore, be attributed to the small extent of the pursuit movement. Rather, it seems that the differences in motion cancellation between pursuit and saccade must be attributed to differences in the extraretinal signals that accompany them.

The Filehne illusion itself can be taken as evidence for a difference between the effects of the pursuit ERS and 
the saccadic ERS. Even though the background movement seen during the Filehne illusion is not perceived by all Os and has a paradoxical nature, it represents a clear contrast to the perception of the background during a saccade, where no hint of background movement is perceived under normal conditions. The results of Condition II show that this difference cannot be attributed, as Gregory assumed, to the difference in the nature of the image motion; it must, therefore, be attributed to some difference between the pursuit ERS and the saccadic ERS. Somehow, background movement is cancelled or suppressed by the saccadic ERS but not by the pursuit ERS.

The position taken here is that there is a fundamental difference between the signal which causes cancellation during the saccade and that which generates motion during pursuit. It could be argued, however, that the difference is simply one of strength. Such an argument might be made in defense of the assumption of the cancellation theory that a single algebraically summating signal is responsible for all eye-movement phenomena. The reason for the failure of cancellation during pursuit, it would be argued, is that this signal is much weaker during pursuit than during the saccade and simply doesn't have the strength necessary to cause cancellation.

This explanation seems plausible, especially considering the results of Condition I of the present study which indicate that the pursuit ERS seems to take a relatively long time to build to its maximum value. Such an explanation can be rejected, however, on the evidence presented by the Filehne illusion itself. Here, the pursuit ERS has presumably attained its maximum value; it has the power to generate a strong perception of the motion of the pursued object. It should, according to the assumption of algebraic summation, have the power to cancel retinal image motion of similar strength. Its failure to do so is sufficient reason to reject this explanation.

The question of the nature of the ERS causing cancellation during the saccade remains. There seem to be two distinct possibilities. It is possible that the cancellation theory is true only for saccadic eye-movement phenomena and that the saccadic ERS is capable of algebraic summation. This ERS would then be responsible for both cancellation and generation of motion, but only during saccadic movement. Alternatively, the saccadic ERS could be a suppression or blocking signal, simply raising the threshold for apparent motion during the saccade. This latter alternative has been suggested and discussed by Wallach and Lewis (1966), Stoper (1967), and Hochberg (1972).

The chief drawback of a simple suppression signal is its lack of versatility. It can cancel apparent movement but never generate it. Thus, an additional signal must be hypothesized to explain, e.g., the apparent movement of an afterimage during a saccade (Phenomenon 4). The algebraically summating signal was assumed by the cancellation theory because one single signal could explain all five eye-movement phenomena, and this seems clearly preferable and more parsimonious than the assumption of two distinct signals. However, as concluded here, at least two distinct signals are required to explain all five eye-motion phenomena, even if one of them is capable of algebraic summation. The advantage of the assumption of algebraic summation over simple suppression is thereby lost.

The simplest explanation of the five phenomena in terms of two signals is made by assuming that the same signal which generates motion during pursuit (Phenomenon 1) also generates it during the saccade (Phenomena 4 and 5). The cancelling signal which causes perceived stability (Phenomenon 2) thus needs no motion generation capacity and need be no more complex than a simple suppression signal. This suppression signal would presumably not occur during passive eye movement, thus explaining by its absence the apparent motion seen in Phenomenon 3. Whether or not the signal that generates motion during pursuit is really the same one that generates it during the saccade remains, however, an open question.

In summary, the nature of the extraretinal signals accompanying eye movements appear to be as follows: The pursuit extraretinal signal carries magnitude and sign information. It is independent of the retinal signal and does not add to it algebraically. It is capable of generating apparent motion, but not of cancelling it. It generates apparent motion of a stimulus object only after the image of the object has been present on the retina some minimum time, perhaps a time long enough to allow the eye to travel through some minimum extent. A different signal must occur during saccadic movement to bring about perceived stability. This signal could function by simply raising the threshold of motion whenever a saccade occurs, but it would not function during pursuit. The signal that generates apparent motion of an afterimage during the saccade could be essentially the same as the signal that generates motion during pursuit, although there is no clear evidence on this point.

\section{REFERENCES}

Barlow, H. B., \& Hill, R. M. Selective sensitivity to direction of movement in ganglion cells of the rabbit retina. Science, 1963 , $139,412-414$.

Brown, J. F. The visual perception of velocity. Psychologische Forschung, 1931, 14, 199-232.

Bruell, J. H., \& Albee, G. W. Notes toward a motor theory of visual egocentric localization. Psychological Review, 1955, 62, 391-399.

Dodge, $R$. Five types of eye movements in the horizontal meridian plane of the field of regard. American Journal of Physiology, 1903, 8, 307-329.

Duncker, K. Über induzierte Bewegung. Psychologische Forschung, 1929, 12, 180-259.

Filehne. W. Über das optische Warnehmen von Bewegungen. Zeitschrift für Sinnesphysiologic. 1922, 53, 134-145. 
Fleischl, E. Physiologisch-optische Notizen. Sitzgsber. Akad. Wiss. Wien, Abt. III, 1882, 86, 17-25. (Cited in Brown. J. F. 1931)

Gibson, J. J. The perception of the visual world. Boston: Houghton Mifflin, 1950.

Gibson, J. J. The visual perception of objective motion and subjective movement. Psychological Review, 1954. 61. 304-314.

Gibson, J. J. Research on the visual perception of motion and change. Second Symposium on Physiological Psychology, School of Aviation Medicine. Pensacola, Florida, 1958.

Gibson, J. J. The senses considered as perceptual systems. Boston: Houghton Mifflin. 1966.

Gibson, J. J. What gives rise to the perception of motion? Psychological Review, 1968, 75, 335-346.

Gregory, R. L. Eye movements and the stability of the visual world. Nature, 1958, 182, 1214-1216.

Gregory, R. L. Ey'e and brain. New York: McGraw-Hill, 1966.

Gyr, J. W. Is a theory of direct visual perception adequate? Psychological Bulletin, 1972, 77, 246-261.

Helmholtz, H. von. Handbuch der physiologischen Optik. Leipzig: Voss, 1866. (Translated and edited by J. P. C. Southall from 3rd ed., 1925: A treatise on physiological optics. Vol. 3. New York: Dover, 1963.)

Hering, E. Der Raumsinn und die Bewegungen der Auges. In L. Hermann (Ed.), Handbuch der Physiologie. 3 (Part 1), 1879. (English translation edited by C. A. Raddie: Spatial sense and movements of the eve. Baltimore: American Academy of Optometry, 1942.)

Heywood, S., \& Churcher, J. Eye movements and the afterimage. I. Tracking the afterimage. Vision Research, 1971, 11, 1163-1168.

Hochberg, J. Perception. II. Space and movement. In J. W. Kling and L. Riggs (Eds.), Woodworth and Schlosberg's Experimental psychology. New York: Holt, Rinehart, \& Winston, 1972. Pp. 475-546.

Howard, I., \& Templeton, W. Human spatial orientation. New York: Wiley, 1966.

Hubel, D. H., \& Wiesel, T. N. Receptive fields, binocular interaction, and functional architecture' in the cat's visual cortex. Journal of Physiology, 1962, 160, 106-154.

James, W. The principles of psychology. Vol. 2. New York: Holt, 1890.

Johansson, G. Configurations in event perception, an experimental study. Uppsala: Almqvist \& Wicksell, 1950.

Koffka, K. Principles of Gestalt psychology. London: Routledge \& Kegan Paul, 1935.

Mack, A. An investigation of the relationship between eye and retinal image movement in the perception of movement. Perception \& Psychophysics, 1970, 8, 291-298.

Mack, A., \& Bachant, J. Perceived movement of the afterimage during eye movements. Perception \& Psychophysics, 1969, 6, 379-384.

Mack, A., \& Herman, E. Position constancy during pursuit eye movement: An investigation of the Filehne illusion. Quarterly Journal of Experimental Psychology, in press.

Mashhour, M. Psychophysical relations in the perception of velocity. Stockholm: Almqvist \& Wicksell, 1964.

Matin, L., Matin, E., \& Pearce, D. G. Visual perception of direction when voluntary saccades occur: $I$. Relation of visual direction of a fixation target extinguished before a saccade to a flash presented during the saccade. Perception \& Psychophysics, 1969, 5, 65-80.

Matin, L., Matin, E., \& Pola, V. Visual perception of direction when voluntary saccades occur: II. Relation of visual direction of a fixation target extinguished before a saccade to a subsequent test flash presented before the saccade. Perception \& Psychophysics, 1970, 8, 9-14.

Monahan, J. S. Extraretinal feedback and visual localization. Perception \& Psychophysics, 1972, 12, 349-353.

Rashbass. C. The relationship between saccadic and smooth tracking eye movements. Journal of Physiology. 1961. 159. 326-338.

Rock. I.. \& Ebenholtz, S. Stroboscopic movement based on change of phenomenal rather than retinal location. American Journal of Psychology, 1962, 75, 193-207.

Sherrington, C. S. Observations on the sensual role of the proprioceptive nerve supply of the extrinsic ocular muscles. Brain, 1918, 41, 332-343.

Stoper, A. E. Vision during pursuit movement: The role of oculomotor information. (Doctoral dissertation, Brandeis University.) Ann Arbor, Michigan: University Microfilms. 1967. No. 67-16, 579 .

Teuber, H. L. Perception. In J. Field (Ed.), Handbook of physiology-Neurophysiology III. Baltimore: Williams \& Wilkins, 1960. Pp. 1595-1668.

von Holst, E. Relations between the central nervous system and the peripheral organs. A nimal Behaviour, 1954, 2, 89-94.

Walls, G. L. The problem of visual direction. Monograph. American Journal of Optometry, 1951, 28, 55-83, 115-146, 173-212.

Wallach, H. Perception of motion. Scientific American, 1959 , 201, 56-60.

Wallach, H., \& Lewis, C. The effect of abnormal displacements of the retinal image during eye movements. Perception \& Psychophysics, 1965, 1, 25-29.

Westheimer, G. Mechanism of saccadic eye movements. Archives of Ophthalmology, 1954a, 52, 710-724.

Westheimer, G. Eye movement responses to a horizontally moving stimulus. Archives of Ophthalmology, 1954b, 52, 932-941.

\section{NOTES}

1. It has been argued (Mashhour, 1964) that the apparent movement of a pursued object is due to lack of perfect following and the resulting retinal image motion. That this is not the case is evidenced by the fact that an afterimage appears to move during smooth (pursuit) eye movements (Heywood \& Churcher, 1971).

2. A notable exception is Gibson $(1954,1966,1968)$, who considers that "transformations in the stimulus array" contain all the information necessary to determine motion or stability and that a process taking eye movements into account need not be assumed. However, this theory does not seem able to adequately explain Phenomena 3, 4, or 5 above. A recent criticism of Gibson's theory has been made by Gyr (1972).

3. There is one version of the cancellation theory (Hering, 1879; Bruell \& Albee, 1955; Rock \& Ebenholtz, 1962) which is represented by Fig. 1 only with a somewhat loose interpretation of that figure. This version [referred to in a previous paper (Stoper, 1967) as the position cancellation theory] assumes that eye movement is taken into account in determining the perceived position of objects. It further assumes that motion is seen only as a result of a change in perceived position. The extraretinal signal in this theory would function to change the "local sign" of the retinal elements. The retinal signal would carry information as to a change of location of an image on the retina. These two signals would effectively add algebraically, and their "resultant" would be the apparent change in location of a perceived object. A nonzero change in location would presumably give rise to apparent motion of the object in the appropriate direction.

4. Originally suggested by $\mathrm{H}$. Wallach (personal communication).

5. The genrally accepted explanation of this motion analysis phenomenon is in terms of the Gestalt "frame of reference" (Duncker, 1929; Johansson, 1950: Wallach. 1959). The "common" motion of the moving points (in the case of the rolling wheel, the common motion would be the horizontal translatory component) is somehow extracted. and then this 
common motion, or some system possessing this common motion, is used as a frame of reference for the "remaining" motion. The mechanics of this frame of reference is, however, generally left undefined. If the assumption of independence of the pursuit extraretinal signal and the retinal signal is accepted, it follows that one quite natural way of performing motion analysis would be the ocular pursuit of the "common" motion. The retina itself would then serve as a frame of reference for the "remaining" motion. When the eye follows the translatory component of a rolling wheel, the points at the rim will describe a circle relative to the retina. The circular component would thus be carried by the retinal signal, while the translatory component would be carried by the pursuit extraretinal signal. Both these components would remain distinct in perception because of the independence of the two motion signals.

6. If the background of the pursuit movement is a normally illuminated room, most Os agree that the background seems to move, in some sense, but does not change position. This indicates apparently perfect position constancy. Perfect position constancy would indicate $100 \%$ compensation for eye movement, though in this case it seems likely that it comes about simply through the tendency of a large visual stimulus to assume the role of stationary frame of reference (cf. Duncker, 1929). Position compensation during pursuit has been measured (Stoper, 1967; Experiment II), using small briefly presented background stimuli, and found to be almost nonexistent. It would be expected that position constancy would increase with size of the background stimuli, though this was not investigated systematically. The results of a recent experiment, using larger background stimuli (Mack \& Herman, in press), do indicate a much greater degree of position constancy during pursuit. The retrograde motion seen during the Filehne illusion, however, is evidence that even if the background stimulus fills the entire visual field and produces apparently perfect position constancy, many Os will nevertheless report motion of the background.

(Received for publication July 21,1972 ; revision received November $17,1972$.

\section{ERRATUM}

Beck, J., and Stevens, A. An aftereffect to discrete stimuli producing apparent movement and succession. Perception \& Psychophysics, 1972, 12 (6), 482-487.-Page 482, Column 2, line 40, the sentence beginning "An $\mathrm{O}$ was told to fixate. .." should read as follows: "An $\mathrm{O}$ was told to fixate at all times on a red neon light (NE 51), which was constantly in view and was placed 2 in. about the midpoint of the two stimuli. On page 485 , Column 2 , line 23 , should read as follows "It is unclear why In Condition D, with a 2-in. separation, there was a tendency to judge the ISI of the test stimulus as greater." 